\title{
Indecomposable polynomials and their spectrum
}

by

\author{
Arnaud Bodin, Pierre Dèbes and Salah Najib (Lille)
}

1. Introduction. Fix an integer $n \geq 2$ and a $n$-tuple of indeterminates $\underline{x}=\left(x_{1}, \ldots, x_{n}\right)$. A non-constant polynomial $F(\underline{x}) \in k[\underline{x}]$ with coefficients in an algebraically closed field $k$ is said to be indecomposable in $k[\underline{x}]$ if it is not of the form $u(H(\underline{x}))$ with $H(\underline{x}) \in k[\underline{x}]$ and $u \in k[t]$ with $\operatorname{deg}(u) \geq 2$. An element $\lambda^{*} \in k$ is called a spectral value of $F(\underline{x})$ if $F(\underline{x})-\lambda^{*}$ is reducible in $k[\underline{x}]$. It is well-known that

(1) $F(\underline{x}) \in k[\underline{x}]$ is indecomposable if and only if $F(\underline{x})-\lambda$ is irreducible in $\overline{k(\lambda)}[\underline{x}]$ (where $\lambda$ is an indeterminate),

(2) if $F(\underline{x}) \in k[\underline{x}]$ is indecomposable, then the subset $\operatorname{sp}(F) \subset k$ of all spectral values of $F(\underline{x})$ - the spectrum of $F(\underline{x})$-is finite; and in the opposite case, $\operatorname{sp}(F)=k$,

(3) more precisely, if $F(\underline{x}) \in k[\underline{x}]$ is indecomposable and for every $\lambda^{*} \in k, n\left(\lambda^{*}\right)$ is the number of irreducible factors of $F(\underline{x})-\lambda^{*}$ in $k[\underline{x}]$, then $\rho(F):=\sum_{\lambda^{*} \in k}\left(n\left(\lambda^{*}\right)-1\right) \leq \operatorname{deg}(F)-1$. In particular, $\operatorname{card}(\operatorname{sp}(F)) \leq \operatorname{deg}(F)-1$.

Statement (3), which is known as Stein's inequality, is due to Stein [13] in characteristic 0 and Lorenzini [10] in arbitrary characteristic (but for two variables); see [11] for the general case.

This paper offers some new results in this context.

In $\S 2$, given an indecomposable polynomial $F(\underline{x})$ with coefficients in an integral domain $A$ and a ring morphism $\sigma: A \rightarrow k$ with $k$ an algebraically closed field, we investigate the connection between the spectrum of $F(\underline{x})$ and that of the polynomial $F^{\sigma}(\underline{x})$ obtained by applying $\sigma$ to the coefficients of $F(\underline{x})$. Theorem 2.1 provides a conclusion à la Bertini-Noether, which, despite its basic nature, does not seem to be available in the literature: under minimal assumptions on $A$, the connection is the expected one gener-

2000 Mathematics Subject Classification: 12E05, 11C08, $12 \mathrm{Y} 05$.

Key words and phrases: irreducible and indecomposable polynomials, Stein's theorem, spectrum of a polynomial. 
ically. For example if $A=\mathbb{Z}$, "spectrum" and "reduction modulo a prime $p$ " commute if $p$ is suitably large (depending on $F$ ). We give other typical applications, notably for a specialization morphism $\sigma$. Related results are given in [3].

For two variables, we give in $\S 3$ an indecomposability criterion for a reduced polynomial modulo some prime $p$ (Theorem 3.1 ) that is more precise than Theorem 2.1: the condition "for suitably large $p$ " is replaced by some explicit condition on $F(x, y)$ and $p$, possibly satisfied for small primes. This criterion uses some results on good reduction of curves and covers due to Grothendieck, Fulton et al.; we will follow here Zannier's version [14]. Another criterion based on the Newton polygon of a polynomial is given in [4].

$\S 4$ is devoted to the connection between the indecomposability properties over a field $K$ and over its algebraic closure $\bar{K}$. While it has been known they are equivalent in many circumstances, for example in characteristic 0 , it has remained to handle the inseparable case to obtain a definitive conclusion. That is the purpose of Proposition 4.1, which, conjoined with previous works, shows that the only polynomials $F(\underline{x})$ indecomposable in $K[\underline{x}]$ but decomposable in $\bar{K}[\underline{x}]$ are $p$ th powers in $\bar{K}[\underline{x}]$, where $p>0$ is the characteristic of $K$ (Theorem 4.2).

$\S 5$ is aimed at counting the number of indecomposable polynomials of a given degree $d$ with coefficients in the finite field $\mathbb{F}_{q}$. We show that most polynomials are indecomposable: the ratio $I_{d} / N_{d}$ of indecomposables of degree $d$ tends to 1 (as $d \rightarrow \infty$ or as $q \rightarrow \infty$ ), and we give some estimate for the error term $1-I_{d} / N_{d}$. The constants involved in our estimates are explicit. For simplicity we mostly restrict to polynomials in two variables, as calculations become more intricate when $n>2$. We also consider the one variable situation (for which the definition of indecomposability is slightly different, see $\S 4.3)$ with the restriction that $q$ and $d$ are relatively prime. A unified treatment of the general case $n \geq 2$ is offered in a parallel work of von zur Gathen [8], who also considers the "wild case" $(n=1$ with $(q, d) \neq 1)$ in [9].

\section{Spectrum and morphisms}

Notation. If $\sigma: A \rightarrow B$ is a ring morphism, we denote the image of $a \in A$ by $a^{\sigma}$. For $P(\underline{x}) \in A[\underline{x}]$, we denote the polynomial obtained by applying $\sigma$ to the coefficients of $P$ by $P^{\sigma}(\underline{x})$. If $V \subset \mathbb{A}_{A}^{n}$ is the Zariski closed subset associated with a family of polynomials $P_{i}(\underline{x}) \in A[\underline{x}]$, we denote by $V^{\sigma}$ the Zariski closed subset of $\mathbb{A}_{B}^{n}$ associated with the family of polynomials $P_{i}^{\sigma}(\underline{x}) \in B[\underline{x}]$.

If $S \subset A$ is a multiplicative subset such that all elements from $S^{\sigma}$ are invertible in $B$, we still denote by $\sigma$ the natural extension $S^{-1} A \rightarrow B$ of the original morphism $\sigma$. 
Fix an integrally closed ring $A$, with a perfect fraction field $K$.

An effective divisor $D=\sum_{i=1}^{r} n_{i} a_{i}$ of $\bar{K}$ is said to be $K$-rational if the coefficients of the polynomial $P(T)=\prod_{i=1}^{r}\left(T-a_{i}\right)^{n_{i}}$ are in $\left.K{ }^{1}\right)$. A morphism $\sigma: A \rightarrow k$ to an algebraically closed field $k$ is then said to be defined at $D$ if the coefficients of $P(T)$ have a common denominator $d \in A$ such that $d^{\sigma}$ is non-zero in $k\left({ }^{2}\right)$. In this case we denote by $P^{\sigma}(T) \in k[T]$ the image polynomial of $P(T)$ by the morphism $\sigma$ (extended to the fraction field of $A$ with denominators a power of $d$ ) and by $D^{\sigma}$ the effective divisor of $k$ whose support is the set of roots of $P^{\sigma}(T)$ and coefficients are the corresponding multiplicities.

2.1. Statement. For more precision, we use the spectral divisor rather than the spectrum: it is the divisor $\operatorname{spdiv}(F)=\sum_{\lambda^{*} \in k}\left(n\left(\lambda^{*}\right)-1\right) \lambda^{*}$ of the affine line $\mathbb{A}^{1}(k)$. Its support is the spectrum of $F$, and Stein's inequality rewrites $\operatorname{deg}(\operatorname{spdiv}(F)) \leq \operatorname{deg}(F)-1$.

Theorem 2.1. Let $F(\underline{x}) \in A[\underline{x}]$ be indecomposable in $\bar{K}[\underline{x}]$. Then there exists a non-zero element $h_{F} \in A$ such that the following holds. For every morphism $\sigma: A \rightarrow k$ to an algebraically closed field $k$, if $h_{F}^{\sigma} \neq 0$, then $F^{\sigma}(\underline{x})$ is indecomposable in $k[\underline{x}]$, the morphism $\sigma: A \rightarrow k$ is defined at the divisor $\operatorname{spdiv}(F)$, and $\operatorname{spdiv}\left(F^{\sigma}\right)=(\operatorname{spdiv}(F))^{\sigma}$; in particular, $\rho\left(F^{\sigma}\right)=\rho(F)$ and $\operatorname{sp}\left(F^{\sigma}\right)=(\operatorname{sp}(F))^{\sigma}$.

The first stage of the proof will produce the spectrum as a Zariski closed subset of the affine line $\mathbb{A}_{A}^{1}$ over the ring $A$. Specifically the following can be drawn from the proof: there is a proper $\left(^{3}\right)$ Zariski closed subset $V_{F} \subset \mathbb{A}_{A}^{1}$ such that for every morphism $\sigma: A \rightarrow k$ as above,

(*) the polynomial $F^{\sigma}(\underline{x})$, if it is of degree equal to $\operatorname{deg}(F)$, is indecomposable in $k[\underline{x}]$ if and only if the Zariski closed subset $V_{F}^{\sigma} \subset \mathbb{A}_{k}^{1}$ is proper, and in this case $\operatorname{sp}\left(F^{\sigma}\right)=V_{F}^{\sigma}(k)$.

When applied to the inclusion morphism $A \rightarrow \bar{K}$, Theorem 2.1 shows that the spectrum of $F(\underline{x})$ is equal to the Zariski closed subset $V_{F}(\bar{K})$. In particular, it is $K$-rational. The same is true for the spectral divisor of $F(\underline{x})$ as $n\left(\lambda^{\tau}\right)=n(\lambda)$ for each $\lambda \in \bar{K}$ and each $\tau \in \operatorname{Gal}(\bar{K} / K)$.

Making the constant $h_{F}$ from Theorem 2.1 explicit is an interesting next goal. This requires good bounds for the "Noether forms" associated with

$\left({ }^{1}\right)$ Under our hypothesis " $K$ perfect", this amounts to the invariance of $P(T)$, or of $D$, under $\operatorname{Gal}(\bar{K} / K)$.

$\left({ }^{2}\right)$ Under our hypothesis " $A$ integrally closed", this amounts to saying the elements $a_{i}$ themselves have a common denominator $d \in A$ (that is, $d a_{i}$ is integral over $A, i=1, \ldots, r$ ) such that $d^{\sigma} \neq 0$.

$\left({ }^{3}\right)$ That is, distinct from the whole surrounding space (here the affine line $\mathbb{A}_{A}^{1}$ over the ring $A$ ); equivalently, there exists a non-zero polynomial in the associated ideal. 
the polynomial $F(\underline{x})-\lambda \in \overline{K(\underline{\lambda})}[\underline{x}]$ in $\S 2.3 .1$. Some work of Busé, Chèze and Najib in this direction is in progress [3].

\subsection{Typical applications}

2.2.1. Situation 1. If $A=\mathbb{Z}$, then $h_{F} \in \mathbb{Z}, h_{F} \neq 0$. Theorem 2.1, applied with $\sigma: \mathbb{Z} \rightarrow \overline{\mathbb{F}}_{p}$ the reduction morphism modulo a prime number $p$ yields:

for all suitably large $p$, the reduced polynomial $\bar{F}(\underline{x})$ modulo $p$ is indecomposable in $\overline{\mathbb{F}}_{p}[\underline{x}]$ and its spectral divisor is obtained by reducing that of $F(\underline{x})$, that is, $\operatorname{spdiv}(\bar{F})=\overline{\operatorname{spdiv}(F)}$.

2.2.2. Situation 2 . Take $A=k[t]$ with $k$ an algebraically closed field and $\underline{t}=\left(t_{1}, \ldots, t_{r}\right)$ some indeterminates. Denote by $F(\underline{t}, \underline{x})$ the polynomial $F(\underline{x})$ of the general statement. Theorem 2.1, applied with $\sigma$ the specialisation morphism $k[\underline{t}] \rightarrow k$ that maps $\underline{t}=\left(t_{1}, \ldots, t_{r}\right)$ to an $r$-tuple $\underline{t}^{*}=\left(t_{1}^{*}, \ldots, t_{r}^{*}\right) \in k^{r}$ yields:

for all $\underline{t}^{*}$ off a proper Zariski closed subset of $k^{r}$, the specialized polynomial $F\left(\underline{t}^{*}, \underline{x}\right)$ is indecomposable in $k[\underline{x}]$ and its spectral divisor is obtained by specializing that of $F(\underline{t}, \underline{x})$.

2.2.3. Situation 3. $F(\underline{x})$ is the generic polynomial in $n$ variables and of degree $d$. Take for $A$ the ring $\mathbb{Z}\left[a_{i}\right]$ generated by the indeterminates $a_{i}$ corresponding to the coefficients of $F(\underline{x})$; the multi-index $\underline{i}=\left(i_{1}, \ldots, i_{n}\right)$ ranges over the set $I_{n, d}$ of all $n$-tuples of integers $\geq 0$ such that $i_{1}+\cdots+i_{n} \leq d$.

Classically the polynomial $F(\underline{x})$ is irreducible in $\overline{\mathbb{Q}\left(a_{i}\right)}[\underline{x}]$, hence it is indecomposable. Theorem 2.1, applied with $\sigma: A \rightarrow k$ a specialization morphism of the $a_{\underline{i}}$, shows that all polynomials $f(\underline{x}) \in k[\underline{x}]$ of degree $d$ are indecomposable except possibly those from the proper Zariski closed subset corresponding to the equation $h_{F}=0$ (with $h_{F}$ viewed in $k\left[a_{i}\right]$ ).

For polynomials $f(\underline{x})$ outside the closed subset $h_{F}=0$, the spectrum of $f$ is obtained by specializing the generic spectrum. However, we have:

Proposition 2.2. For $d>2$ or $n>2$, the generic spectrum is empty. For $d=n=2$, it contains a single element, given by

$$
a_{00}-\frac{a_{02} a_{10}^{2}+a_{20} a_{01}^{2}-a_{01} a_{10} a_{11}}{4 a_{02} a_{20}-a_{11}^{2}} .
$$

For $d>2$ or $n>2$, polynomials with a non-empty spectrum lie in the Zariski closed subset $h_{F}=0$.

Proof. Assume that the generic spectrum is not empty. If $k$ is an algebraically closed field and $\mathcal{R}_{n, d}$ (resp. $\left.\mathcal{P}_{n, d}\right|_{a_{0}=0}$ ) denotes the set of polynomials $P(\underline{x}) \in k[\underline{x}]$ of degree $\leq d$ that are reducible in $k[\underline{x}]$ (resp. whose constant term is zero), the correspondence $P(\underline{x}) \mapsto P(\underline{x})-P(\underline{0})$ induces an algebraic morphism $\left.\mathcal{R}_{n, d} \rightarrow \mathcal{P}_{n, d}\right|_{a_{0}=0}$ which is generically surjective (that 
is, surjective above a non-empty Zariski open subset of $\left.\left.\mathcal{P}_{n, d}\right|_{a_{0}=0}\right)$. It follows that $\mathcal{R}_{n, d}$ is of codimension $\leq 1$ in the space $\mathcal{P}_{n, d}$ of all polynomials in $k[\underline{x}]$ of degree $\leq d$. This observation provides the desired conclusion in the case $n=2$ and $d>2$ : indeed $\operatorname{codim}_{\mathcal{P}_{2, d}}\left(\mathcal{R}_{2, d}\right)=d-1$ [7, Theorem 2].

For $d=n=2$, the equation " $(u x+a y+b)(v x+c y+d)=F(x, y)$ modulo the constant term" with unknowns $u, a, b, v, c, d$ is readily solved: reduce to the case $a_{20}=u=v=1$, find the unique solution for the 4-tuple $(a, b, c, d)$ and compute $b d$; the generic spectral value is then $a_{00}-b d$.

Finally assume that for $d \geq 2$ and $n>2$, there exists a generic spectral value $\lambda \in \bar{K}$ (with $K=\mathbb{Q}\left(a_{\underline{i}}\right)$ ). Let $F(\underline{x})-\lambda=Q(\underline{x}) R(\underline{x})$ be a non-trivial factorization in $\bar{K}[\underline{x}]$. Specializing $x_{3}, \ldots, x_{n}$ to 0 gives a non-trivial factorization in $\bar{K}\left[x_{1}, x_{2}\right]$ of the generic polynomial of degree $d$ in two variables. From the first part of the proof, we have $d=2$. Furthermore, the above case provides the necessary value of $\lambda$. Now specializing $x_{2}$ and $x_{4}, \ldots, x_{n}$ to 0 leads to a different value. This is a contradiction.

\subsection{Proof of Theorem 2.1}

2.3.1. 1st stage: elimination theory. This stage is aimed at showing Proposition 2.3 below, which generalizes the Bertini-Noether theorem [6, Prop. 9.4.3]. It is proved in the general situation

(Hyp) a polynomial $\mathcal{F}(\underline{\lambda}, \underline{x}) \in A[\underline{\lambda}, \underline{x}]$ is irreducible in $\overline{K(\underline{\lambda})}[\underline{x}]$, where $\underline{\lambda}=\left(\lambda_{1}, \ldots, \lambda_{s}\right)$ is an s-tuple of indeterminates $(s \geq 0)$.

We will use it in the special case $\mathcal{F}(\underline{\lambda}, \underline{x})=F(\underline{x})-\lambda$. The hypotheses " $A$ integrally closed" and " $K$ perfect" are not necessary for this stage.

As in Situation 3, consider some indeterminates $\left(a_{\underline{i}}\right)_{\underline{i} \in I_{n, d}}$ corresponding to the coefficients of a polynomial of degree $d$ in $n$ variables. A polynomial with coefficients in a ring $R$ corresponds to a morphism $\phi: \mathbb{Z}\left[a_{\underline{i}}\right] \rightarrow R$; denote by $F\left(a_{\underline{i}}^{\phi}\right)(\underline{x}) \in A[\underline{x}]$ the corresponding polynomial. Let $\varphi_{\underline{\lambda}}: \mathbb{Z}\left[a_{\underline{i}}\right] \rightarrow A[\underline{\lambda}]$ be the morphism corresponding to the polynomial from statement (Hyp): $\mathcal{F}(\underline{\lambda}, \underline{x})=F\left(a_{\underline{i}}^{\varphi^{\lambda}}\right)(\underline{x})$.

From Noether's theorem [12, $\S 3.1$, Theorem 32], there exist finitely many universal homogeneous forms $\mathcal{N}_{h}\left(a_{\underline{i}}\right)(1 \leq h \leq D=D(n, d))$ in the $a_{\underline{i}}$ and with coefficients in $\mathbb{Z}$ such that:

(4) for every morphism $\phi: \mathbb{Z}\left[a_{\underline{i}}\right] \rightarrow k$ to an algebraically closed field $k$, the polynomial $F\left(a_{\underline{i}}^{\phi}\right)(\underline{x})$, if it is of degree $d$, is reducible in $k[\underline{x}]$ if and only if $\mathcal{N}_{h}\left(a_{\underline{i}}^{\phi}\right)=0$ for $h=1, \ldots, D$.

For $\phi$ taken to be the morphism $\varphi_{\underline{\lambda}}: \mathbb{Z}\left[a_{\underline{i}}\right] \rightarrow A[\underline{\lambda}] \subset \overline{K(\underline{\lambda})}$, the elements $\mathcal{N}_{h}\left(a_{\underline{i}}^{\varphi^{\underline{\lambda}}}\right) \in A[\underline{\lambda}]$ are polynomials $N_{h}(\underline{\lambda})$. Let $V_{\mathcal{F}} \subset \mathbb{A}_{A}^{s}$ be the Zariski closed subset corresponding to the ideal they generate; it is a proper closed subset. Indeed, as $\mathcal{F}(\underline{\lambda}, \underline{x})$ is irreducible in $\overline{K(\underline{\lambda})}[\underline{x}]$, from (4), at least one 
of the polynomials $N_{h}(\underline{\lambda})$, say $N_{h_{0}}(\underline{\lambda})$, is non-zero. Denote by $a_{\mathcal{F}} \in A$ the product of a non-zero coefficient of $N_{h_{0}}(\underline{\lambda})$ and the non-zero coefficient of some monomial of $\mathcal{F}(\underline{\lambda}, \underline{x})$ of degree $d$ in $\underline{x}$.

If $R$ is an integral domain and $\Sigma: A[\underline{\lambda}] \rightarrow R$ a morphism, then (4), with $\phi$ taken to be $\Sigma \circ \varphi_{\lambda}: \mathbb{Z}\left[a_{i}\right] \rightarrow R \hookrightarrow \kappa$ and $\kappa=\overline{\operatorname{Frac}(R)}$, shows that the polynomial $\mathcal{F}^{\Sigma} \in R[\underline{x}]$, if of degree $d$, is irreducible in $\kappa[\underline{x}]$ if and only if at least one of the elements $N_{h}^{\Sigma} \in R$ is non-zero (note that $\mathcal{F}^{\Sigma}=F\left(a_{\underline{i}}^{\Sigma \varphi_{\underline{\lambda}}}\right)(\underline{x})$ and $\left.\mathcal{N}_{h}\left(a_{\underline{i}}^{\Sigma \varphi_{\underline{\lambda}}}\right)=\mathcal{N}_{h}\left(a_{\underline{i}}^{\varphi^{\underline{\lambda}}}\right)^{\Sigma}\right)$, or, equivalently, if the corresponding Zariski closed subset of $\operatorname{Spec}(R)$ is proper.

Let $\sigma: A \rightarrow k$ be a morphism with $k$ algebraically closed. Apply the above first with $\Sigma$ taken to be the morphism $\sigma: A[\underline{\lambda}] \rightarrow k[\underline{\lambda}]$ and then, for $\underline{\lambda}^{*} \in k^{s}$, with $\Sigma$ taken to be the morphism $s_{\underline{\lambda}^{*}} \circ \sigma: A[\underline{\lambda}] \rightarrow k$ obtained by composing $\sigma: A[\underline{\lambda}] \rightarrow k[\underline{\lambda}]$ with the specialization morphism $s_{\underline{\lambda}^{*}}: k[\underline{\lambda}] \rightarrow k$ to $\underline{\lambda}^{*}$. We conclude:

Proposition 2.3 (Bertini-Noether generalized).

(a) The polynomial $\mathcal{F}^{\sigma}(\underline{\lambda}, \underline{x})$, if it is of degree $d$ in $\underline{x}$, is irreducible in $\overline{k(\underline{\lambda})}[\underline{x}]$ if and only if the Zariski closed subset $V_{\mathcal{F}}^{\sigma} \subset \mathbb{A}_{k}^{s}$ is proper. All these conditions are satisfied if $a_{\mathcal{F}}^{\sigma}$ is non-zero in $k$.

(b) If the polynomial $\mathcal{F}^{\sigma}\left(\underline{\lambda}^{*}, \underline{x}\right)$ is of degree $d$, then it is reducible in $k[\underline{x}]$ if and only if $\underline{\lambda}^{*}$ is in the set $V_{\mathcal{F}}^{\sigma}(k)$.

2.3.2. 2nd stage: implications for the spectrum of $F(\underline{x})$. We return to the situation where $\mathcal{F}(\lambda, \underline{x})=F(\underline{x})-\lambda$. Denote the Zariski closed subset $V_{\mathcal{F}}$ from $\S 2.3 .1$ by $V_{F}$; it is a Zariski closed subset of the affine line $\mathbb{A}_{A}^{1}$. The preceding conclusions, conjoined with the connection, recalled in $\S 1$, between indecomposability of $F(\underline{x})$ and irreducibility of $F(\underline{x})-\lambda$, yield statement $(*)$ from $\S 2.1$.

Denote by $s_{F}(\lambda)$ the g.c.d. of the polynomials $N_{h}(\lambda)$ in the ring $K[\lambda]$. Write it as $s_{F}(\lambda)=S_{F}(\lambda) / c_{1}$ with $S_{F}(\lambda) \in A[\lambda]$ and $c_{1} \in A$ non-zero. The polynomial $S_{F}(\lambda)$ is non-zero and its distinct roots in $\bar{K}$, say $\lambda_{1}, \ldots, \lambda_{s}$, which are the common roots in $\bar{K}$ of the polynomials $N_{h}(\lambda)$, are the spectral values of $F(\underline{x})$ (note that $F(\underline{x})-\lambda^{*}$ is of degree $d$ for all $\lambda^{*} \in \bar{K}$ ). Thus we have $S_{F}(\lambda)=c_{2} \prod_{i=1}^{s}\left(\lambda-\lambda_{i}\right)^{n_{i}} \in A[\lambda]$ for some exponents $n_{i}>0$ and $c_{2} \in A, c_{2} \neq 0$. It follows that the set $\operatorname{sp}(F)=\left\{\lambda_{1}, \ldots, \lambda_{s}\right\}$ is $K$-rational. As already noted, the same is then true for the spectral divisor $\operatorname{spdiv}(F)$.

2.3.3. 3rd stage: invariance of the spectrum of $F$ via morphisms . Fix a morphism $\sigma: A \rightarrow k$ with $k$ algebraically closed. Denote by $a_{F}$ the element $a_{\mathcal{F}}$ from $\S 2.3 .1$ for $\mathcal{F}=F(\underline{x})-\lambda$. If $a_{F}^{\sigma} \neq 0$, then $F^{\sigma}(\underline{x})$ is of degree $d$ and indecomposable in $k[\underline{x}]$. Furthermore, its spectral values are the roots in $k$ of the g.c.d. of the polynomials $N_{h}^{\sigma}(\lambda)$. 
Note that the element $c_{2}$ above is a common denominator of $\lambda_{1}, \ldots, \lambda_{s}$; if $c_{2}^{\sigma} \neq 0$, the morphism $\sigma: A \rightarrow k$ is defined at $\operatorname{spdiv}(F)$.

Lemma 2.4. There exists $c_{3} \in A, c_{3} \neq 0$, such that, if $a_{F}^{\sigma} c_{1}^{\sigma} c_{2}^{\sigma} c_{3}^{\sigma} \neq 0$, the polynomial $S_{F}^{\sigma}(\lambda) \in k[\lambda]$ equals (up to some non-zero multiplicative constant in $k)$ the g.c.d. in $k[\lambda]$ of the polynomials $N_{h}^{\sigma}(\lambda)(1 \leq h \leq D)$. In particular, $\operatorname{sp}\left(F^{\sigma}\right)=(\operatorname{sp}(F))^{\sigma}$.

Proof. The problem is whether the g.c.d. commutes with $\sigma$. The Euclidean algorithm provides the g.c.d. as the last non-zero remainder. To reach our goal, it suffices to guarantee that for each division $a=b q+r$ in $K[\lambda]$ involved in the algorithm, the identity $a^{\sigma}=b^{\sigma} q^{\sigma}+r^{\sigma}$, with $\sigma$ suitably extended, be the division of $a^{\sigma}$ by $b^{\sigma}$ in $k[\lambda]$. For this, write $a, b, q$ and $r$ in the form $n(\lambda) / m$ with $n(\lambda) \in A[\lambda]$ and $m \in A$, consider the product $\beta$ of denominators $m$ of $a, b, q$ and $r$ with the coefficients of highest degree monomials in the numerators $n(\lambda)$ of $b$ and $r$ and require that $\beta^{\sigma} \neq 0$. Multiplying all elements $\beta$ for all divisions leading to the g.c.d. of two, then of all polynomials in question, leads to a non-zero element $c_{3} \in A$ which satisfies the desired statement.

REMARK 2.5. Morphisms and g.c.d. do not commute in general: for example $\operatorname{gcd}(\lambda, \lambda+a)$ is 1 generically, but equals $\lambda$ if $a=0$.

2.3.4. 4th stage: invariance of $\operatorname{spdiv}(F)$ via morphisms. It remains to extend the conclusion " $\mathrm{sp}\left(F^{\sigma}\right)=(\operatorname{sp}(F))^{\sigma}$ " to the spectral divisor $\operatorname{spdiv}(F)$. We will show how to guarantee that, via the morphism $\sigma$, the spectral values remain distinct and the associated decompositions of $F(\underline{x})-\lambda$ have the same numbers of distinct irreducible factors $\left({ }^{4}\right)$.

Consider the discriminant of the polynomial $\prod_{i=1}^{s}\left(\lambda-\lambda_{i}\right)$; it is a nonzero element of $K$. Write it as $c_{4} / c_{5}$ with $c_{4}, c_{5} \in A$, non-zero. If $c_{4}^{\sigma} c_{5}^{\sigma} \neq 0$, the polynomials $S_{F}(\lambda)$ and $S_{F}^{\sigma}(\lambda)$ have the same number of distinct roots, whence $\operatorname{card}\left(\operatorname{sp}\left(F^{\sigma}\right)\right)=\operatorname{card}\left((\operatorname{sp}(F))^{\sigma}\right)=\operatorname{card}(\operatorname{sp}(F))$.

For $i=1, \ldots, s$, let $F(\underline{x})-\lambda_{i}=\prod_{j=1}^{n\left(\lambda_{i}\right)} Q_{i j}(\underline{x})^{k_{i j}}$ be a factorization (into distinct irreducible polynomials) in $\bar{K}[\underline{x}]$. Let $E / K$ be a finite Galois extension that contains the finite set $\mathcal{C}$ of all coefficients involved in all above factorizations, $c_{6}$ be a non-zero element of $A$ such that $c_{6} c$ is integral over $A$ for all $c \in \mathcal{C}$, and $c_{7}$ be the discriminant of a basis of $E$ over $K$ the elements of which are integral over $A$. Denote by $B$ the fraction ring of $A$ with denominator a power of $c_{6} c_{7}$, and by $B_{E}^{\prime}$ the integral closure of $B$ in $E$. The ring $B_{E}^{\prime}$ is a free $B$-module of rank $[E: K]$. Assume that $c_{6}^{\sigma} c_{7}^{\sigma} \neq 0$. The morphism $\sigma: A \rightarrow k$ extends to a morphism $B \rightarrow k$, and, as $k$ is

$\left({ }^{4}\right)$ The argument will also show the degrees of these irreducible factors, say $Q_{\lambda, j}$, remain the same and thus so does the quantity $\min _{\lambda \in \operatorname{sp}(F)}\left(\sum_{j} \operatorname{deg}\left(Q_{\lambda, j}\right)-1\right)$, which replaces $\operatorname{deg}(F)-1$ in Lorenzini's refined version [10] of Stein's inequality. 
algebraically closed, this morphism $\sigma: B \rightarrow k$ can in turn be extended to a morphism $\widetilde{\sigma}: B_{E}^{\prime} \rightarrow k$.

The polynomials $Q_{i j}(\underline{x})$ are in the ring $B_{E}^{\prime}[\underline{x}]$ and are absolutely irreducible. The (classical) Bertini-Noether theorem provides a non-zero element $\beta \in B_{E}^{\prime}$ such that, if $\beta^{\widetilde{\sigma}} \neq 0$, then each of the polynomials $Q_{i j}^{\widetilde{\sigma}}(\underline{x})$ is absolutely irreducible. Therefore the decomposition $F^{\sigma}(\underline{x})-\lambda_{i}^{\widetilde{\sigma}}=\prod_{j=1}^{n\left(\lambda_{i}\right)} Q_{i j}^{\widetilde{\sigma}}(\underline{x})$ obtained from the preceding one by applying $\widetilde{\sigma}$, is the factorization of $F^{\sigma}(\underline{x})-\lambda_{i}^{\widetilde{\sigma}}$ into irreducible polynomials in $k[\underline{x}]$.

It remains to ensure that for $i$ fixed, the polynomials $Q_{i j}^{\widetilde{\sigma}}(\underline{x})$ are different, even up to non-zero multiplicative constants. For any two (distinct) polynomials $Q_{i j}(\underline{x}), Q_{i j^{\prime}}(\underline{x})$, the matrix with rows the tuples of coefficients of the two polynomials has a $2 \times 2$-block with a non-zero determinant. Denote the product of all such determinants for all possible couples $\left(Q_{i j}(\underline{x}), Q_{i j^{\prime}}(\underline{x})\right)$ by $\delta$; it is a non-zero element of $B_{E}^{\prime}$. Denote then by $\nu$ the norm of $\beta \delta$ relative to the extension $E / K$. As $A$ is integrally closed, so is $B$ and $\nu \in B$. Write it as $\nu=c_{8} /\left(c_{6} c_{7}\right)^{\gamma}$ with $c_{8} \in A$ and $\gamma \in \mathbb{N}$. Condition $c_{6}^{\sigma} c_{7}^{\sigma} c_{8}^{\sigma} \neq 0$ implies $\beta_{F}^{\widetilde{\sigma}} \delta_{F}^{\widetilde{\sigma}} \neq 0$. Theorem 2.1 is finally established for $h_{F}=a_{F} \prod_{i=1}^{8} c_{i}$.

REMARK 2.6. The same proof, with the polynomial $\mathcal{F}(\underline{\lambda}, \underline{x})$ from $\S 2.3 .1$ of the form $F(\underline{x})-\lambda G(\underline{x})$ with $F(\underline{x}), G(\underline{x}) \in A[\underline{x}]$ and $\operatorname{deg} G \leq \operatorname{deg} F$, leads to the more general form of Theorem 2.1 for which indecomposable polynomials are replaced by indecomposable rational functions (in this case, "indecomposable" means not of the form $u(H(\underline{x}))$ with $H(\underline{x})$ and $u(t)$ rational functions and $\left.\operatorname{deg}(u) \geq 2\left({ }^{5}\right)\right)$. A spectral value of a rational function $F(\underline{x}) / G(\underline{x})$ is an element $\lambda$ such that the polynomial $F(\underline{x})-\lambda G(\underline{x})$ is reducible. Statements (1)-(3) from $\S 1$ remain true, except that the bound in Stein's inequality should be replaced by $(\operatorname{deg}(F))^{2}-1$ [2], [10]. More generally, one can take $\mathcal{F}(\underline{\lambda}, \underline{x})$ of the form $F(\underline{x})-\lambda_{1} G_{1}(\underline{x})-\cdots-\lambda_{s} G_{s}(\underline{x})$ with $F(\underline{x}), G_{1}(\underline{x}), \ldots, G_{s}(\underline{x}) \in A[\underline{x}]$ and handle other situations studied in the literature.

3. An indecomposability criterion modulo $p$. In this section $n=2$, $A$ is a Dedekind domain and its fraction field $K$ is assumed to be of characteristic 0 . Fix also a non-zero prime ideal $\mathfrak{p}$ of $A$ and assume its residue field $k=A / \mathfrak{p}$ is of characteristic $p>0$. Denote by $\widetilde{x}$ the image of an element $x$ under the reduction morphism $A \rightarrow k$. The situation " $A=\mathbb{Z}$ and $\mathfrak{p}=p \mathbb{Z}$ " is typical.

Let $F(x, y) \in A[x, y]$ be an indecomposable polynomial in $\bar{K}[x, y]$ of degree $d \geq 1$, monic in $y$.

$\left({ }^{5}\right)$ The degree of a rational function is the maximum of the degrees of its numerator and denominator. 
Here is our strategy to guarantee indecomposability of $F(x, y)$ modulo $\mathfrak{p}$. Pick $\lambda^{*} \in A \backslash \operatorname{sp}(F)$ (using Stein's theorem, this can be done for $\lambda^{*}$ not too large). Thus $F(x, y)-\lambda^{*}$ is irreducible in $\bar{K}[x, y]$. It follows from the classical Bertini-Noether theorem that if "p is large enough", then the reduced polynomial $F(x, y)-\lambda^{*}$ modulo $\mathfrak{p}$ is absolutely irreducible. Therefore $F(x, y)$ is indecomposable modulo $\mathfrak{p}$ (as there is at least one non spectral value). However, the constants involved in the condition "p large enough" are too large for a practical algorithmic use. We will follow an alternative approach, based on good reduction criteria for covers, and more precisely Zannier's criterion [14].

Consider the discriminant with respect to $y$ of $F(x, y)-\lambda$ :

$$
\Delta_{F}(x, \lambda)=\operatorname{disc}_{y}(F(x, y)-\lambda) .
$$

Denote then the product of all distinct irreducible factors of $\Delta_{F}(x, \lambda)$ in $K(\lambda)[x]$ by $\Delta_{F}^{\mathrm{red}}(x, \lambda)$; more precisely, $\Delta_{F}^{\mathrm{red}}(x, \lambda)$ is defined by the following formula, which is also algorithmically more practical:

$$
\Delta_{F}^{\mathrm{red}}(x, \lambda)=c(\lambda) \frac{\Delta_{F}(x, \lambda)}{\operatorname{gcd}\left(\Delta_{F}(x, \lambda),\left(\Delta_{F}\right)_{x}^{\prime}(x, \lambda)\right)}
$$

where the g.c.d. is calculated in the ring $K(\lambda)[x]$ (using the Euclidean algorithm for example) and $c(\lambda) \in K(\lambda)$ is the rational function, defined up to some invertible element in $A$, that makes $\Delta_{F}^{\text {red }}(x, \lambda)$ a primitive polynomial in $A[\lambda][x]$. Consider next the polynomial

$$
\Delta_{F}(\lambda)=\operatorname{disc}_{x}\left(\Delta_{F}^{\mathrm{red}}(x, \lambda)\right) .
$$

We have $\Delta_{F}(\lambda) \in A[\lambda]$ and $\Delta_{F}(\lambda) \neq 0$. Finally, let $\Delta_{0}(\lambda) \in A[\lambda]$ be the coefficient of the highest monomial in $\Delta_{F}(x, \lambda)$ (viewed in $A[\lambda][x]$ ).

Theorem 3.1. Assume, in addition to $F(x, y)$ being indecomposable in $\bar{K}[x, y]$, that the reduced polynomial $\widetilde{\Delta}_{0}(\lambda) \widetilde{\Delta}_{F}(\lambda)$ is non-zero in $k[\lambda]$ and that $p>\operatorname{deg}_{y}(F)$. Then $\widetilde{F}(x, y)$ is indecomposable in $\bar{k}[x, y]$.

The assumption $p>\operatorname{deg}_{y}(F)$ can be replaced by the weaker condition that $p$ does not divide the order of the Galois group of $F(x, y)-\lambda$, viewed as a polynomial in $\overline{K(\lambda)}(x)$ (see footnote 8 ).

The assumptions of Theorem 3.1 may not be sufficient to guarantee the extra conclusions $\operatorname{sp}(\widetilde{F})=\widetilde{\operatorname{sp}(F)}$ and $\operatorname{spdiv}(\widetilde{F})=\widetilde{\operatorname{spdiv}(F)}$ from Theorem 2.1 (which may not even be well-defined). They, however, still imply that if $V_{F} \subset \mathbb{A}_{A}^{1}$ is the Zariski closed subset from $\S 2.1$, then the reduced

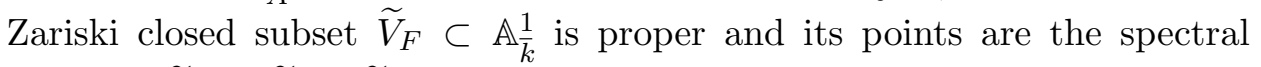
values of $\widetilde{F}: \operatorname{sp}(\widetilde{F})=\widetilde{V}_{F}(\bar{k})$. 
REMARK 3.2 (an indecomposability test). Theorem 3.1 provides the following procedure to decide whether a non-constant polynomial $F(x, y) \in$ $\mathbb{Q}[x, y]$ is indecomposable.

One may assume that $F(x, y) \in \mathbb{Z}[x, y]$ and $\operatorname{deg}_{y}(F)>0$. Up to changing $y$ to $a y$ for some $a \in \mathbb{Z}$, one may also reduce to the case of $F(x, y)$ monic with respect to the reverse lexicographic order (for which $y>x$ ). Observe that the polynomial $\Delta_{F}(x, \lambda)=\operatorname{disc}_{y}(F(x, y)-\lambda)$ is non-zero in general (whether $F$ is indecomposable or not): indeed, none of the roots $y$ of $(\partial F / \partial y)(x, y)$, which are in $\overline{\mathbb{Q}(x)}$, can also be a root of $F(x, y)-\lambda$. Consequently, the polynomial $\Delta_{0}(\lambda) \Delta_{F}(\lambda)$ is non-zero in general. Pick a prime $p$ satisfying the assumptions of Theorem 3.1: $p>\operatorname{deg}_{y}(F)$ and $\widetilde{\Delta}_{0}(\lambda) \widetilde{\Delta}_{F}(\lambda)$ is non-zero in $\mathbb{F}_{p}[\lambda]$. Test for decomposability of $\widetilde{F}(x, y)$ (from $\S 4$, it is sufficient to only consider decompositions over $\mathbb{F}_{p}$ instead of $\left.\overline{\mathbb{F}}_{p}\right)$. If $\widetilde{F}(x, y)$ is decomposable, then $F(x, y)$ is decomposable by Theorem 3.1. As we explain below, the converse also holds: if $\widetilde{F}(x, y)$ is indecomposable, then $F(x, y)$ is indecomposable.

Namely, if $F(x, y)$ is decomposable then $F(x, y)$ has a non-trivial decomposition $F(x, y)=u(H(x, y))$ with $u$ and $H$ with coefficients in $\overline{\mathbb{Q}}$ and even in $\mathbb{Q}$ (see $\S 4$ ). Furthermore, $F(x, y) \in \mathbb{Z}[x, y]$ being monic forces these coefficients to be in $\mathbb{Z}$. For one variable, this is explained in [5], and in general one can reduce to this case thanks to a Kronecker substitution. Specifically, one may assume that $H(x, y)$ is monic (with respect to the same order), and then so is $u$, and that $H(0,0)=0$. Write $F\left(x, x^{m}\right)=u\left(H\left(x, x^{m}\right)\right)$ with $m$ large enough to have $F\left(x, x^{m}\right)$ and $H\left(x, x^{m}\right)$ monic. From [5, Theorem 2], $u(t)$ and $H\left(x, x^{m}\right)$ must have integral coefficients, and consequently so does $H(x, y)$ (for $m \gg 1)$. Finally, reduction modulo $p$ of $F(x, y)=u(H(x, y))$ provides a non-trivial decomposition of $\widetilde{F}(x, y)$.

Proof of Theorem 3.1. The prime ideal $\mathfrak{p} \subset A$ determines a discrete valuation $v$ of $K$ whose valuation ring is the localized ring $A_{\mathfrak{p}}$; the fraction field of $A_{\mathfrak{p}}$ and its residue field remain equal to $K$ and $k$ respectively. The hypotheses and conclusions from Theorem 3.1 are unchanged if $A$ is replaced by $A_{\mathfrak{p}}$. The valued field $(K, v)$ can then also be replaced by any finite extension of the completion $K_{v}$, and $A$ by the new valuation ring; the discrete valuation $v$ uniquely extends, the residue field is replaced by some (finite) extension of $k$, the indecomposability properties of $F(x, y)$ over $K$ or over $K_{v}$ are equivalent.

Thus we may and will assume that $(K, v)$ is a complete discretely valued field, that $A$ is its valuation ring (which is integrally closed) and that the field $K$ and the residue field $k$ contain as many (finitely many) algebraic elements over the original fields as necessary. 
The polynomial $\Delta_{F}(x, \lambda)$ is in $A[x, \lambda]$ and its factorization into irreducible polynomials in $K(\lambda)[x]$ can be written

$$
\Delta_{F}(x, \lambda)=\delta_{0}(\lambda) \prod_{i=1}^{s} \Delta_{i}(x, \lambda)^{\alpha_{i}}
$$

where the polynomials $\Delta_{i}(x, \lambda)$ are in $A[x, \lambda]$, irreducible in $K(\lambda)[x]$, pairwise distinct (even up to some constant in $K$ ), and primitive in $A[\lambda][x]$, where $\delta_{0}(\lambda) \in A[\lambda]$ and where the $\alpha_{i}$ are positive integers. Then, up to some invertible element in $A$, we have

$$
\Delta_{F}^{\mathrm{red}}(x, \lambda)=\prod_{i=1}^{s} \Delta_{i}(x, \lambda) .
$$

Also note that the polynomial $\Delta_{0}(\lambda)$ is a multiple in $A[\lambda]$ of the product of $\delta_{0}(\lambda)$ and the highest monomial coefficients $\delta_{1}(\lambda), \ldots, \delta_{s}(\lambda)$ of the polynomials $\Delta_{1}(x, \lambda), \ldots, \Delta_{s}(x, \lambda)$ (viewed in $A(\lambda)[x]$ ).

Pick next $\widetilde{\lambda}^{*} \in k$ such that $\widetilde{\Delta}_{0}\left(\widetilde{\lambda}^{*}\right) \widetilde{\Delta}_{F}\left(\widetilde{\lambda}^{*}\right) \neq 0$ in $k$, then lift it to some element $\lambda^{*} \in A$ such that $\lambda^{*} \notin \operatorname{sp}(F)$. This is possible in view of the preliminary remark.

The set of roots of $\Delta_{F}\left(x, \lambda^{*}\right)$ contains the set of finite $\left({ }^{6}\right)$ branch points of the cover of $\mathbb{P}_{x}^{1}\left({ }^{7}\right)$ determined by the (absolutely irreducible) polynomial $F(x, y)-\lambda^{*}$. The preliminary remark makes it possible to assume that these roots are in $K$. Furthermore, as $\widetilde{\delta}_{i}\left(\widetilde{\lambda}^{*}\right) \neq 0$, we have $\delta_{i}\left(\lambda^{*}\right) \in A \backslash \mathfrak{p}, i=$ $1, \ldots, s$; therefore these roots are integral over $A$ and so are in $A$.

As $\Delta_{F}\left(\lambda^{*}\right) \neq 0$, the roots of $\Delta_{F}^{\text {red }}\left(x, \lambda^{*}\right)$ in $\bar{K}$ are distinct, and as $\delta_{0}\left(\lambda^{*}\right) \neq 0$, they are the roots of $\Delta_{F}\left(x, \lambda^{*}\right)$. As $\widetilde{\Delta}_{0}\left(\widetilde{\lambda}^{*}\right) \neq 0, \widetilde{\Delta}_{F}\left(x, \widetilde{\lambda}^{*}\right)$ is not the zero polynomial. As $\widetilde{\Delta}_{F}\left(\widetilde{\lambda}^{*}\right) \neq 0$, the roots of $\widetilde{\Delta}_{F}^{\text {red }}\left(x, \widetilde{\lambda}^{*}\right)$, which are those of the polynomial $\widetilde{\Delta}_{F}\left(x, \widetilde{\lambda}^{*}\right)$, are distinct. Thus we conclude that the distinct roots of the polynomial $\Delta_{F}\left(x, \lambda^{*}\right)$, and a fortiori the branch points of the cover considered above, have distinct reductions modulo the ideal $\mathfrak{p}$.

It follows from standard results on good reduction of covers, and more precisely here, from the main theorem of [14] that, under the assumption $p>\operatorname{deg}_{y}(F)\left({ }^{8}\right), \widetilde{F}(x, y)-\widetilde{\lambda}^{*}$ is absolutely irreducible. Hence $\widetilde{F}(x, y)$ is indecomposable in $\bar{k}[x, y]$.

$\left({ }^{6}\right)$ That is, distinct from the point at infinity.

$\left({ }^{7}\right)$ The subscript " $x$ " indicates that the cover is induced by the correspondence $(x, y) \mapsto x$. In fact, the problem is symmetric in the variables $x$ and $y$ which can be switched in our statement.

$\left(^{8}\right)$ It suffices to assume that $p$ does not divide the order of the Galois group of $F(x, y)-\lambda^{*}$, which divides the order of the Galois group of $F(x, y)-\lambda$, which itself divides $\left(\operatorname{deg}_{y}(F)\right)$ !. 


\section{Indecomposability over $K$ versus $\bar{K}$}

4.1. Statements (for $n \geq 2$ variables). The indecomposability property over an algebraically closed field, which we recalled in $\S 1$, can in fact be defined over an arbitrary field: just require that the polynomials $u(t)$ and $H(\underline{x})$ involved have their coefficients in the field in question. The results below identify the only cases where the property is not the same over some field $K$ and over some extension $E$. The following result handles the case where $E / K$ is purely inseparable, which was missing in the literature.

Proposition 4.1. Let $E / K$ be a purely inseparable algebraic field extension of characteristic $p>0$ and $F(\underline{x}) \in K[\underline{x}]$. Assume $F(\underline{x})$ is not of the form $b G(\underline{x})^{p}+c$ with $G(\underline{x}) \in E[\underline{x}]$ and $b, c \in K$. Then $F(\underline{x})$ is indecomposable in $K[\underline{x}]$ if and only if it is indecomposable in $E[\underline{x}]$.

If $E=\bar{K}$, the assumption on $F(\underline{x})$ merely says that $F(\underline{x})$ is not a $p$ th power in $\bar{K}[\underline{x}]$, which in turn is equivalent to at least one exponent in $F(\underline{x})$ not being a multiple of $p$. Clearly this assumption cannot be removed: for example, if $\alpha \in \bar{K} \backslash K$ but $\alpha^{p}=a \in K$ then $x^{p}+a y^{p}$ is indecomposable in $K[\underline{x}]$ but decomposable in $\bar{K}[\underline{x}]$.

In [1, Proposition 1], Arzhantsev and Petravchuk show the equivalence from Proposition 4.1 without any assumption on $F(\underline{x})$, but in the case of a separable extension $E / K$ (possibly of positive transcendence degree). As any extension is a purely inseparable algebraic extension of some separable extension, conjoining their result with ours shows that, under the assumption on $F(\underline{x})$ from Proposition 4.1, the equivalence holds for an arbitrary extension $E / K$. We can be more precise.

Theorem 4.2. Let $E / K$ be a field extension and $F(\underline{x}) \in K[\underline{x}]$ be a non-constant polynomial. Then the following are equivalent:

(i) $F(\underline{x})$ is indecomposable in $K[\underline{x}]$ but decomposable in $E[\underline{x}]$.

(ii) (a) $K$ is of characteristic $p>0$ and $E / K$ is inseparable,

(b) $F(\underline{x})=b G(\underline{x})^{p}+c$ for some $G(\underline{x}) \in E[\underline{x}]$ and $b, c \in K$,

(c) $G(\underline{x})^{p}$ is indecomposable in $K[\underline{x}]$.

Condition (ii)(c) implies that $G(\underline{x})$ is not of the form $u(H(\underline{x}))$ with $u \in E[t], H(\underline{x}) \in E[\underline{x}], \operatorname{deg}(u) \geq 2$ and both $u(t)^{p} \in K[t]$ and $H(\underline{x})^{p} \in K[\underline{x}]$. But there are other possible polynomials that should be excluded whose description is more intricate.

\subsection{Proofs}

Proof of Proposition 4.1. The converse part is obvious. For the direct part, assume $F(\underline{x})$ is decomposable in $E[\underline{x}]$. Then it is decomposable over some finite extension of $K$ contained in $E$, which admits a finite system of generators $\alpha_{1}, \ldots, \alpha_{s}$ with irreducible polynomial over $K$ of the form 
$x^{p^{n}}-a$ with $a \in K$. The multiplicativity of the degree and of the separable degree imply that the extensions $K\left(\alpha_{1}, \ldots, \alpha_{j+1}\right) / K\left(\alpha_{1}, \ldots, \alpha_{j}\right)$ are purely inseparable for $j=1, \ldots, s-1$. By induction one reduces to the case $s=1$, and then a new induction reduces to the case $E=K(\alpha)$ with $\alpha^{p}=a$ $\in K \backslash K^{p}$.

Assume $F(\underline{x})=h(G(\underline{x}))$ with $h(t) \in K(\alpha)[t]$ such that $\operatorname{deg}(h) \geq 2$ and $G(\underline{x}) \in K(\alpha)[\underline{x}]$. We deduce

$$
F(\underline{x})^{p}={ }^{p} h\left(G(\underline{x})^{p}\right)
$$

where, if $h(t)=\sum_{i=0}^{\operatorname{deg}(h)} h_{i} t^{i}$, we set ${ }^{p} h(t)=\sum_{i=0}^{\operatorname{deg}(h)} h_{i}^{p} t^{i}$. As ${ }^{p} h(t) \in K[t]$ and $G(\underline{x})^{p} \in K[\underline{x}]$ (since $y^{p} \in K$ for all $y \in K(\alpha)$ ), this shows that the field $K\left(F(\underline{x}), G(\underline{x})^{p}\right)$ is of transcendence degree 1 over $K$. From Gordan's Theorem [12, $\S 1.2$, Th. 3], there exists $\theta(\underline{x}) \in K(\underline{x})$ such that

$$
K\left(F(\underline{x}), G(\underline{x})^{p}\right)=K(\theta(\underline{x})) .
$$

Furthermore from $[12, \S 1.2$, Th. 4$]$, one may assume that $\theta(\underline{x}) \in K[\underline{x}]$. Thus

$$
\begin{cases}F(\underline{x})=u(\theta(\underline{x})) & \text { with } u(t) \in K(t), \\ G(\underline{x})^{p}=v(\theta(\underline{x})) & \text { with } v(t) \in K(t) .\end{cases}
$$

As $F(\underline{x})$ and $G(\underline{x})^{p}$ are polynomials, $u(t), v(t)$ are necessarily in $K[t]$. It follows from the indecomposability of $F(\underline{x})$ over $K$ that $\operatorname{deg}(u)=1$, which gives $G(\underline{x})^{p}=w(F(\underline{x}))$ for some $w \in K[t]$. But then $G(\underline{x})^{p}=w \circ h(G(\underline{x}))$, which, since $G(\underline{x})$ is non-constant, amounts to $T^{p}=w \circ h(T)$ where $T$ is an indeterminate. $\operatorname{As} \operatorname{deg}(h) \geq 2$ and $p$ is a prime, we have $\operatorname{deg}(w)=1$ and $\operatorname{deg}(h)=p$, which gives $F(\underline{x})=b G(\underline{x})^{p}+c$ for some $b, c \in K$.

Note that because of the inductive process, the conclusion " $b, c \in K$ " should really be that $b, c$ are in the first subfield of the initial reduction. But $F(\underline{x})$ being in $K[\underline{x}]$ then implies that $b \gamma^{p} \in K$ for some non-zero $\gamma \in E$ and $b G(\underline{0})^{p}+c \in K$. Up to changing $G(\underline{x})$ to $\gamma^{-1} G(\underline{x})-\gamma^{-1} G(\underline{0})$, one can indeed conclude that $b, c \in K$ in the general situation.

Proof of Theorem 4.2. (i) $\Rightarrow$ (ii): If $K_{s} / K$ is the maximal separable extension contained in $E$, then, from the Arzhantsev-Petravchuk result, $F(\underline{x})$ is indecomposable in $K_{s}[\underline{x}]$. In particular $E \neq K_{s}$, which gives (ii)(a). Proposition 4.1 then provides condition (ii)(b) except that $b$ and $c$ are a priori in $K_{s}$, but using again the final note of the proof of Proposition 4.1, one can indeed choose $b, c \in K$. Condition (ii)(c) then readily follows from (ii)(b) and the indecomposability of $F(\underline{x})$ in $K[\underline{x}]$. The other implication (ii) $\Rightarrow$ (i) is clear.

4.3. One variable. In Proposition $4.1, F(\underline{x})$ is a polynomial in two variables or more. In one variable, the indecomposability definition should be modified (for otherwise it is trivial): a polynomial $F(x) \in k[x]$ is said to be 
indecomposable in $k[x]$ if it is not of the form $u(H(x))$ with $H(x) \in k[x]$ and $u \in k[t]$ with $\operatorname{deg}(u) \geq 2$ and $\operatorname{deg}(H) \geq 2$.

Proposition 4.3. The conclusion of Proposition 4.1 holds for one variable polynomials.

Proof. The same proof can be used as for Proposition 4.1. It leads to

$$
\begin{cases}F(x)=u(\theta(x)) & \text { with } u(t) \in K[t] \\ G(x)^{p}=v(\theta(x)) & \text { with } v(t) \in K[t] .\end{cases}
$$

But from the indecomposability of $F(\underline{x})$ over $K$, we now deduce that $\operatorname{deg}(u)$ $=1$ or $\operatorname{deg}(\theta)=1$.

The case $\operatorname{deg}(u)=1$ is handled as before. In the other case, we deduce from $\operatorname{deg}(\theta)=1$ that $K\left(F(x), G(x)^{p}\right)=K(x)$, which implies that $K(\alpha)\left(h(G(x)), G(x)^{p}\right)=K(\alpha)(x)$ and so that

$$
K(\alpha)(x) \subset K(\alpha)(G(x)),
$$

which forces $\operatorname{deg}(G)=1$ and contradicts the decomposability assumption in one variable made at the beginning of the proof.

5. Counting indecomposable polynomials over finite fields. For each integer $d \geq 1$, denote the number of polynomials in $\mathbb{F}_{q}[\underline{x}](\underline{x}=$ $\left.\left(x_{1}, \ldots, x_{n}\right)\right)$ of degree $d$ by $N_{d}$. We have

$$
N_{d}= \begin{cases}\left(q^{\left(\begin{array}{c}
n+d-1 \\
n-1
\end{array}\right)}-1\right) \cdot q^{\left(\begin{array}{c}
n+d-1 \\
n
\end{array}\right)} & \text { for general } n, \\
q^{(d+1)(d+2) / 2}\left(1-q^{-d-1}\right) & \text { for } n=2, \\
(q-1) q^{d} & \text { for } n=1 .\end{cases}
$$

Denote the number of those polynomials which are indecomposable (resp. decomposable) by $I_{d}$ (resp. $D_{d}$ ). We have $N_{d}=I_{d}+D_{d}$.

We will study separately the case of $n \geq 2$ variables $(\S 5.1-\S 5.4)$ and the case $n=1(\S 5.5)$.

5.1. Main result. From $\S 5.1$ to $\S 5.4$, we assume $n \geq 2$.

THEOREM 5.1.

(a) $I_{d} / N_{d}$ tends to 1 when $d \rightarrow \infty$ with $q$ fixed, and when $q \rightarrow \infty$ with $d$ fixed.

(b) If $d$ is a product of at most two prime numbers $p \leq p^{\prime}$, then either

- $d=p$ and $D_{d}=q^{d}\left(q^{n}-1\right)$, or

- $d=p^{2}$ and $D_{d}=q^{p-1} N_{p}+\left(q^{d}-q^{2 p-1}\right)\left(q^{n}-1\right)$, or

- $d=p p^{\prime}$ with $p<p^{\prime}$ and

$$
D_{d}=q^{p-1} N_{p^{\prime}}+q^{p^{\prime}-1} N_{p}+\left(q^{d}-2 q^{p+p^{\prime}-1}\right)\left(q^{n}-1\right) .
$$


(c) Assume $n=2$. If $d$ is the product of at least three primes, then

$$
\left|\frac{D_{d}}{N_{d}}-\alpha_{d}\right| \leq \alpha_{d} \beta_{d} \quad \text { where }\left\{\begin{array}{l}
\alpha_{d}=\frac{q^{\ell-1+(d / \ell+1)(d / \ell+2) / 2}}{q^{(d+1)(d+2) / 2}}, \\
\beta_{d}=\frac{d}{q^{d / \ell}},
\end{array}\right.
$$

and $\ell>1$ is the first (hence prime) divisor of $d$.

A version of statement (c) in the general case of $n$ variables is given in [8]. For $n=2$, his first order estimate for $D_{d} / N_{d}$ is the same as ours, that is, $\alpha_{d}$; his error term is improved by a factor $O(q)$.

5.2. An induction formula. Let $K$ be an arbitrary field. Let $F=u \circ H$ be a decomposition of $F \in K[\underline{x}]$ with $u \in K[t], \operatorname{deg} u \geq 2$, and $H \in K[\underline{x}]$. We say that $F=u \circ H$ is a normalized decomposition if $H$ is indecomposable, monic (i.e. the coefficient of the leading term of a chosen order is 1) and its constant term equals zero. Given a decomposition $F=u \circ H$, there exists an associated normalized decomposition $F=u^{\prime} \circ H^{\prime}$. The following lemma shows it is unique.

Lemma 5.2. Let $F=u \circ H=u^{\prime} \circ H^{\prime}$ be two normalized decompositions of $F \in K[\underline{x}]$. Then $u=u^{\prime}$ and $H=H^{\prime}$.

Proof. It follows from $u(H)-u^{\prime}\left(H^{\prime}\right)=0$ that $H$ and $H^{\prime}$ are algebraically dependent over $K$. By Gordan's theorem $[12, \S 1.2$, Theorems 3 and 4] (already used in $\S 4.2$ ), there exists a polynomial $\theta(\underline{x}) \in K[\underline{x}]$ such that $K[\theta]=K\left[H, H^{\prime}\right]$. That is, there exist $v, v^{\prime} \in K[t]$ such that $H=v(\theta)$ and $H^{\prime}=v^{\prime}(\theta)$. As the two decompositions of $F$ are normalized, $H$ and $H^{\prime}$ are indecomposable, so $\operatorname{deg} v=\operatorname{deg} v^{\prime}=1$, and so using the other normalization conditions, we obtain $H=H^{\prime}$. Finally, it follows from $u(H)=u^{\prime}(H)$ that $u=u^{\prime}$.

COROLlary 5.3 (induction formula). With notation as in $\S 5.1$, we have

$$
I_{d}=N_{d}-\sum_{d^{\prime} \mid d, d^{\prime}<d} q^{d / d^{\prime}-1} \times I_{d^{\prime}} .
$$

Proof. Let $d^{\prime} \geq 1$ be a divisor or $d$. There are $(q-1) q^{d / d^{\prime}}$ polynomials $u \in$ $\mathbb{F}_{q}[t]$ of degree $d / d^{\prime}$ and $I_{d^{\prime}} / q(q-1)$ normalized indecomposable polynomials $H \in \mathbb{F}_{q}[\underline{x}]$ of degree $d^{\prime}$. The formula follows, as from Lemma 5.2, every polynomial $F$ counted by $D_{d}$ can be uniquely written as $F=u \circ H$ with $u$ and $H$ as above for some integer $d^{\prime}$ such that $d^{\prime} \mid d, d^{\prime}<d$.

Combined with $I_{1}=N_{1}=q\left(q^{n}-1\right)$ this formula provides an algorithm to compute $I_{d}$ and $D_{d}$, which is convenient for small $d$. 
5.3. Proof of Theorem 5.1(a) and (b). The formulas in (b) directly follow from Corollary 5.3. If $d=p$ is a prime number, we have $D_{p}=q^{p-1} I_{1}=$ $q^{p-1} N_{1}=q^{p}\left(q^{n}-1\right)$. If $d=p^{2}$ then

$$
D_{d}=q^{p-1} I_{p}+q^{p^{2}-1} I_{1}=q^{p-1}\left(N_{p}-q^{p}\left(q^{n}-1\right)\right)+q^{p^{2}}\left(q^{n}-1\right) .
$$

Computations are similar for $d=p p^{\prime}$. To prove (a) we write

$$
N_{d}-I_{d}=D_{d}=\sum_{d^{\prime} \mid d, d^{\prime}<d} q^{d / d^{\prime}} I_{d^{\prime}} \leq \sum_{d^{\prime} \mid d, d^{\prime}<d} q^{d / d^{\prime}} N_{d^{\prime}} .
$$

The sum has at most $d$ terms and each is $\leq q^{d} N_{d / 2}$, whence

$$
1-\frac{I_{d}}{N_{d}} \leq d q^{d} \frac{N_{d / 2}}{N_{d}},
$$

and the announced result follows, as the right-hand side term tends to 0 in the two situations considered in the statement of Theorem 5.1(a).

5.4. Proof of Theorem 5.1(c). In this subsection we assume that $n=2$ and that $d$ has at least three prime divisors.

5.4.1. A technical lemma

Lemma 5.4. Let $b(d)=\frac{1}{2}(d+1)(d+2)$. Let $\ell>1$ be the first divisor of $d$ and $\ell^{\prime}>\ell$ be the second divisor of $d$. Let $\lambda \geq \ell^{\prime}$ be a divisor of $d$ and $\ell^{\prime \prime}>1$ be the first divisor of $d / \ell$. Then

(1) $b\left(d / \ell^{\prime}\right)+\ell^{\prime} \geq b(d / \lambda)+\lambda$,

(2) $b(d / \ell)+\ell-d / \ell \geq b\left(d / \ell^{\prime}\right)+\ell^{\prime}$,

(3) $b(d / \ell)+1-d / \ell \geq b\left(d / \ell \ell^{\prime \prime}\right)+\ell^{\prime \prime}$.

Proof. (1) We have

$$
b\left(d / \ell^{\prime}\right)+\ell^{\prime}-b(d / \lambda)-\lambda=\frac{1}{2}\left(\frac{d}{\ell^{\prime}}-\frac{d}{\lambda}\right)\left(\frac{d}{\ell^{\prime}}+\frac{d}{\lambda}+3-2 \frac{\ell^{\prime} \lambda}{d}\right) \geq 0,
$$

because $d / \ell^{\prime}-d / \lambda \geq 0$ and

$$
\frac{d}{\ell^{\prime}}+\frac{d}{\lambda}+3-2 \frac{\ell^{\prime} \lambda}{d} \geq \frac{d}{\ell^{\prime}}+4-2 \ell^{\prime} \geq 0,
$$

as $d$ has at least three prime divisors.

(2) We have $\ell \ell^{\prime} \leq d$ so $\ell^{\prime}-\ell \leq d / \ell$. Moreover, $d / \ell^{\prime} \leq d / \ell-2$ and for all $d \geq 6$ we have $b\left(d / \ell^{\prime}\right) \leq b(d / \ell-2)$. Hence

$$
b(d / \ell)-b\left(d / \ell^{\prime}\right)+\ell-\ell^{\prime}-d / \ell \geq b(d / \ell)-b(d / \ell-2)-2 d / \ell=1 .
$$

(3) If we set $\delta=d / \ell$ then

$$
\begin{aligned}
b(\delta)+1-\delta-b\left(\delta / \ell^{\prime \prime}\right)-\ell^{\prime \prime}= & \frac{1}{2}\left(\delta-\frac{\delta}{\ell^{\prime \prime}}\right)\left(\delta+\frac{\delta}{\ell^{\prime \prime}}-2\right) \\
& +\frac{1}{2}\left(3 \delta-5 \frac{\delta}{\ell^{\prime \prime}}-2 \ell^{\prime \prime}+2\right) .
\end{aligned}
$$


Now $\delta-\delta / \ell^{\prime \prime} \geq 0, \delta+\delta / \ell^{\prime \prime}-2 \geq 0$ and as $\delta$ has at least two prime divisors, it follows that $u\left(\ell^{\prime \prime}\right)=3 \delta-5 \delta / \ell^{\prime \prime}-2 \ell^{\prime \prime}+2 \geq u(2)=\delta / 2-2 \geq 0$.

5.4.2. An upper bound for $D_{d}$. Using the notation of Lemma 5.4, we have

$$
\begin{aligned}
D_{d} & =q^{\ell-1} I_{d / \ell}+\sum_{\lambda \mid d, \lambda>\ell} q^{\lambda-1} I_{d / \lambda} \quad(\text { Corollary 5.3) } \\
& \leq q^{\ell-1} N_{d / \ell}+\sum_{\lambda \mid d, \lambda>\ell} q^{\lambda-1} N_{d / \lambda} \\
& \leq q^{b(d / \ell)+\ell-1}\left(1-\frac{1}{q^{d / \ell+1}}\right)+\sum_{\lambda \mid d, \lambda>\ell} q^{\lambda-1} q^{b(d / \lambda)} \quad\left(\text { explicit formula for } N_{d / \lambda}\right) \\
& \leq q^{b(d / \ell)+\ell-1}\left(1-\frac{1}{q^{d / \ell+1}}\right)+(d-1) q^{b\left(d / \ell^{\prime}\right)+\ell^{\prime}-1} \quad(\text { Lemma } 5.4(1)) \\
& \leq q^{b(d / \ell)+\ell-1}\left(1-\frac{1}{q^{d / \ell+1}}\right)\left(1+\frac{d}{q^{b(d / \ell)-b\left(d / \ell^{\prime}\right)+\ell-\ell^{\prime}}}\right) \\
& \leq q^{b(d / \ell)+\ell-1}\left(1-\frac{1}{q^{d / \ell+1}}\right)\left(1+\frac{d}{q^{d / \ell}}\right) \quad\left(\text { because } \frac{d-1}{1-q^{-d / \ell-1}} \leq d\right)
\end{aligned}
$$

5.4.3. A lower bound for $D_{d}$. Start from $D_{d} \geq q^{\ell-1} I_{d / \ell}$. Then use $\S 5.4 .2$ (or the formulas already proved from Theorem 5.1(b)) to bound $I_{d / \ell}=$ $N_{d / \ell}-D_{d / \ell}$ from below. We obtain

$$
\begin{aligned}
D_{d} \geq & q^{\ell-1} \times\left(q^{b(d / \ell)}\left(1-\frac{1}{q^{d / \ell+1}}\right)\right. \\
& \left.-q^{b\left(d / \ell \ell^{\prime \prime}\right)+\ell^{\prime \prime}-1}\left(1-\frac{1}{q^{d / \ell \ell^{\prime \prime}+1}}\right)\left(1+\frac{d / \ell}{q^{d / \ell \ell^{\prime \prime}}}\right)\right) \\
\geq & q^{\ell-1}\left(1-\frac{1}{q^{d / \ell+1}}\right)\left(q^{b(d / \ell)}-2 q^{b\left(d / \ell \ell^{\prime \prime}\right)+\ell^{\prime \prime}-1}\right) \quad\left(\text { because } \frac{d / \ell}{q^{d / \ell \ell^{\prime \prime}}} \leq 1\right) \\
= & q^{\ell-1}\left(1-\frac{1}{q^{d / \ell+1}}\right) q^{b(d / \ell)}\left(1-\frac{2}{q^{b(d / \ell)-b\left(d / \ell \ell^{\prime \prime}\right)+1-\ell^{\prime \prime}}}\right) \\
\geq & q^{b(d / \ell)+\ell-1}\left(1-\frac{1}{q^{d / \ell+1}}\right)\left(1-\frac{2}{q^{d / \ell}}\right) \quad(\text { Lemma 5.4 }(3)) .
\end{aligned}
$$


5.4.4. Final estimate for $D_{d} / N_{d}$. The upper and lower bounds for $D_{d}$ yield the inequalities

$$
\begin{aligned}
\frac{q^{b(d / \ell)+\ell-1}}{q^{b(d)}} \times \frac{1-q^{-d / \ell-1}}{1-q^{-d-1}} & \times\left(1-\frac{2}{q^{d / \ell}}\right) \leq \frac{D_{d}}{N_{d}} \\
& \leq \frac{q^{b(d / \ell)+\ell-1}}{q^{b(d)}} \times \frac{1-q^{-d / \ell-1}}{1-q^{-d-1}} \times\left(1+\frac{d}{q^{d / \ell}}\right),
\end{aligned}
$$

a little more precise than the announced statement.

5.5. One variable. Here we assume $n=1$. For polynomials in one variable, we use the definition of indecomposability given in $\S 4.3$.

\subsubsection{Main result}

TheOREm 5.5. Assume $q$ and $d$ are relatively prime.

(a) If $d$ is a product of at most two prime numbers $p \leq p^{\prime}$, then either

- $d=p$ and $D_{d}=0$, or

- $d=p^{2}$ and $D_{d}=\frac{q-1}{q} q^{2 p}$, or

- $d=p p^{\prime}$ with $p<p^{\prime}$ and

$$
2 \frac{q-1}{q} q^{p+p^{\prime}}-q^{5} \leq D_{d} \leq 2 \frac{q-1}{q} q^{p+p^{\prime}} .
$$

(b) Assume $d$ is the product of at least three prime numbers. Let $\ell>1$ be the first divisor of $d$ and $\ell^{\prime}>\ell$ be its second divisor. Then

$$
\begin{aligned}
& \frac{d}{2 \ell} \frac{1}{q^{d / \ell-d / \ell^{2}-\ell+1}} \leq \frac{D_{d}}{N_{d}}-\alpha_{d} \leq \frac{d-2}{2 q^{\ell+d / \ell-\ell^{\prime}-d / \ell^{\prime}}} \\
& \text { where } \alpha_{d}=\frac{2}{q^{d-\ell-d / \ell+1}} .
\end{aligned}
$$

As a consequence, $I_{d} / N_{d}$ tends to 1 when $d \rightarrow \infty$ with $q$ fixed, and $q \rightarrow \infty$ with $d$ fixed.

Theorem 5.5 fails if the assumption $(q, d)=1$ is removed. For example for $q=2$ and $d$ even one can compute that $D_{d} / N_{d} \sim 3 \cdot 2^{-d / 2}$ while $\alpha_{d}=4 \cdot 2^{-d / 2}$ in this case.

From now on we assume $q$ and $d$ are relatively prime. The rest of the paper is devoted to the proof of Theorem 5.5. Our strategy is similar to the one used for $n \geq 2$. We view the set $\mathcal{D}_{d}$ of all decomposable polynomials $f(x) \in \mathbb{F}_{q}[x]$ of degree $d$ as the union of smaller sets which we will estimate. More specifically, we write

$$
\mathcal{D}_{d}=\bigcup_{\lambda \mid d, \ell \leq \lambda \leq d / \ell} \mathcal{D}_{\lambda, d / \lambda}
$$

where $\mathcal{D}_{\lambda, d / \lambda} \subset \mathcal{D}_{d}$ is the subset of all $f(x)$ which admit a decomposition $f=u \circ v$ with $u, v \in \mathbb{F}_{q}[x], \operatorname{deg} u=\lambda \geq 2, \operatorname{deg} v=d / \lambda \geq 2, v$ monic and 
of constant term equal to 0 . A difference with the case $n \geq 2$ is that we do not have a partition.

5.5.2. 1st stage: upper bounds. (Assumption $(q, d)=1$ is not used in this subsection.) For every divisor $\lambda \geq 1$ of $d$, denote the cardinality of $\mathcal{D}_{\lambda, d / \lambda}$ by $D_{\lambda, d / \lambda}$. We have

$$
D_{\lambda, d / \lambda} \leq N_{\lambda} \frac{N_{d / \lambda}}{q(q-1)}=\frac{q-1}{q} q^{\lambda+d / \lambda} .
$$

If $\ell>1$ is the first divisor of $d$ and $\ell^{\prime}>\ell$ the second divisor, we have

$$
D_{d} \leq \sum_{\lambda \mid d, \ell \leq \lambda \leq d / \ell} D_{\lambda, d / \ell} \leq \frac{q-1}{q} \sum_{\lambda \mid d, \ell \leq \lambda \leq d / \ell} q^{\lambda+d / \lambda} .
$$

The idea is that the main contribution comes from $D_{\ell, d / \ell}$ and $D_{d / \ell, \ell}$.

If $d$ is the product of exactly two prime numbers $\ell$ and $d / \ell$, then these are the only contributions and we have the desired upper bound. Otherwise we write $\lambda+d / \lambda \leq \ell^{\prime}+d / \ell^{\prime}$ to bound the extra terms and obtain

$$
D_{d} \leq \frac{q-1}{q} q^{\ell+d / \ell}\left(2+\frac{d-2}{q^{\ell+d / \ell-\ell^{\prime}-d / \ell^{\prime}}}\right)
$$

which yields all announced upper bounds in Theorem 5.5. We also deduce this practical bound:

$$
D_{d} \leq d \frac{q-1}{q} q^{\ell+d / \ell}
$$

$\left(\right.$ as $\left.\ell+d / \ell-\ell^{\prime}-d / \ell^{\prime} \geq 1\right)$.

5.5.3. 2nd stage: uniqueness results. We will use Ritt's theorems to control the number of possible decompositions of a given polynomial.

Proposition 5.6. Let $K$ be a field and $f \in K[x]$ be a polynomial of degree $d>0$. Assume the characteristic $p$ of $K$ does not divide $d$. Suppose we have two decompositions $f=u \circ v=u^{\prime} \circ v^{\prime}$ of $f$ with

- $u, v, u^{\prime}, v^{\prime}$ indecomposable,

- $\operatorname{deg} u=\operatorname{deg} u^{\prime} \geq 2, \operatorname{deg} v=\operatorname{deg} v^{\prime} \geq 2$,

- $v, v^{\prime}$ monic with a zero constant term.

Then $u=u^{\prime}$ and $v=v^{\prime}$.

Proof. This follows from the first Ritt theorem [12, §1.3, Theorem 7], which more generally describes in which cases an equality $G_{1} \circ \cdots \circ G_{r}=$ $H_{1} \circ \cdots \circ H_{s}$ with $G_{i}, H_{j}$ indecomposable of degree $>1$ may hold.

As an immediate consequence, we obtain the case $d=p^{2}$ of Theorem 5.5(a): namely, we have $D_{p^{2}}=D_{p, p}=\frac{q-1}{q} q^{2 p}$. 
5.5.4. 3rd stage: lower bounds for $D_{d / \ell, \ell}$ and $D_{\ell, d / \ell}$.

Lemma 5.7. Assume $d$ is not a prime number. Then

$$
D_{\ell, d / \ell} \geq \frac{q-1}{q} q^{\ell+d / \ell}\left(1-\frac{d / \ell}{q^{d / \ell-d / \ell^{2}-\ell+1}}\right),
$$

and the same inequality holds with $D_{\ell, d / \ell}$ replaced by $D_{d / \ell, \ell}$.

Proof. We only give the proof for $D_{\ell, d / \ell}$ as computations for $D_{d / \ell, \ell}$ are the same. In $\mathcal{D}_{\ell, d / \ell}$ we will only count those polynomials $f$ which decompose as $f=u \circ v$ with $u$ and $v$ as in Proposition 5.6. Then we obtain

$$
\begin{aligned}
D_{\ell, d / \ell} & \geq \frac{1}{q(q-1)} I_{\ell} \cdot I_{d / \ell} \\
& \geq \frac{1}{q(q-1)} N_{\ell}\left(N_{d / \ell}-D_{d / \ell}\right) \quad\left(D_{\ell}=0 \text { as } \ell \text { is prime }\right) \\
& =\frac{1}{q(q-1)}(q-1) q^{\ell}\left((q-1) q^{d / \ell}-D_{d / \ell}\right) \\
& =\frac{q-1}{q} q^{\ell+d / \ell}\left(1-\frac{D_{d / \ell}}{(q-1) q^{d / \ell}}\right) .
\end{aligned}
$$

If $d$ is the product of exactly two primes then $D_{d / \ell}=0$ and

$$
D_{\ell, d / \ell} \geq \frac{q-1}{q} q^{\ell+d / \ell},
$$

which in this case is better than the announced result.

If $d$ is the product of at least three primes, use the practical upper bound for $D_{d}$ obtained in Stage 1 to write $D_{d / \ell} \leq \frac{d}{\ell} \frac{q-1}{q} q^{\ell+d / \ell^{2}}$ and deduce

$$
\begin{aligned}
D_{\ell, d / \ell} \geq \frac{q-1}{q} q^{\ell+d / \ell}\left(1-\frac{(d / \ell) \frac{q-1}{q} q^{\ell+d / \ell^{2}}}{(q-1) q^{d / \ell}}\right) & \\
& =\frac{q-1}{q} q^{\ell+d / \ell}\left(1-\frac{d / \ell}{q^{d / \ell-d / \ell^{2}-\ell+1}}\right) .
\end{aligned}
$$

5.5.5. Estimating the multiple decompositions. Next we write

$$
D_{d} \geq \operatorname{card}\left(\mathcal{D}_{\ell, d / \ell} \cup \mathcal{D}_{d / \ell, \ell}\right)=D_{\ell, d / \ell}+D_{d / \ell, \ell}-\operatorname{card}\left(\mathcal{D}_{\ell, d / \ell} \cap \mathcal{D}_{d / \ell, \ell}\right) .
$$

In order to estimate $D_{d}$ we need to estimate the intersection.

Lemma 5.8. We have

$$
\left\{\begin{array}{l}
\operatorname{card}\left(\mathcal{D}_{\ell, d / \ell} \cap \mathcal{D}_{d / \ell, \ell}\right) \leq \frac{d}{\ell} q^{d / \ell^{2}+2 \ell-1} \\
D_{d} \geq 2 \frac{q-1}{q} q^{\ell+d / \ell}\left(1-\frac{2 d}{\ell} \frac{1}{q^{d / \ell-d / \ell^{2}-\ell+1}}\right) .
\end{array}\right.
$$


The lower bound for $D_{d}$ is the remaining inequality to be proved in Theorem 5.5(b). The more precise inequality $(* *)$ in the proof below will complete the proof of Theorem 5.5(a) in the special case $d=p p^{\prime}$.

Proof of Lemma 5.8. (a) If $\operatorname{gcd}(\ell, d / \ell)=1$ then $\operatorname{card}\left(\mathcal{D}_{\ell, d / \ell} \cap \mathcal{D}_{d / \ell, \ell}\right) \leq q^{5}$.

Indeed, let $f \in \mathcal{D}_{\ell, d / \ell} \cap \mathcal{D}_{d / \ell, \ell}$ and let $f=u \circ v$ be a decomposition with $\operatorname{deg} u=\ell$ and $\operatorname{deg} v=d / \ell$. We follow Ritt's second theorem (see $[12, \S 1.4$, Theorem 8$]$ and the notation there). The hypotheses of that result are satisfied because the derivative $u^{\prime}$ of $u$ is non-zero; otherwise $f^{\prime}=0$, and so $f \in \mathbb{F}_{q}\left[x^{p}\right]$ and the characteristic $p$ of $\mathbb{F}_{q}$ divides $d=\operatorname{deg} f$. In the first case of Ritt's second theorem we have $L_{1} \circ u=x^{r} P(x)^{n}$ and $v \circ L_{2}=x^{n}$ (where $r \geq 0, P \in \mathbb{F}_{q}[x]$ and $L_{1}, L_{2}$ are linear functions). In our situation we get $n=d / \ell$ and $\ell=r+(d / \ell) \operatorname{deg} P$. Then $\operatorname{deg} P=\left(\ell^{2}-\ell r\right) / d \leq \ell^{2} / d<1$, so $\operatorname{deg} P=0, L_{1} \circ u=x^{\ell}$ and $v \circ L_{2}=x^{d / \ell}$. Considering all possible linear functions yields at most $(q-1)^{2} q^{2}$ such decompositions. In the second case of Ritt's second theorem we have $L_{1} \circ u=D_{m}\left(x, a^{n}\right)$ and $v \circ L_{2}=D_{n}(x, a)$, $a \in \mathbb{F}_{q}$ (where $D_{n}(x, a)$ denote Dickson's polynomials). We here obtain $m=\ell$ and $n=d / \ell$. Considering all possible linear functions and all $a \in \mathbb{F}_{q}$ yields at most $(q-1)^{2} q^{3}$ such decompositions. Finally, we obtain

$$
\operatorname{card}\left(\mathcal{D}_{\ell, d / \ell} \cap \mathcal{D}_{d / \ell, \ell}\right) \leq(q-1)^{2} q^{2}+(q-1)^{2} q^{3} \leq q^{5} .
$$

(b) If $\operatorname{gcd}(\ell, d / \ell) \neq 1$ then $\operatorname{card}\left(\mathcal{D}_{\ell, d / \ell} \cap \mathcal{D}_{d / \ell, \ell}\right) \leq \frac{d}{\ell} q^{d / \ell^{2}+2 \ell-1}$.

Indeed, let $f \in \mathcal{D}_{\ell, d / \ell} \cap \mathcal{D}_{d / \ell, \ell}$ and let $f=u \circ v$ be a decomposition with $\operatorname{deg} u=\ell$ and $\operatorname{deg} v=d / \ell$. By Ritt's first theorem and because $\operatorname{gcd}(\ell, d / \ell) \neq 1$, either $u$ or $v$ is decomposable. But as $\ell$ is a prime, $\mathcal{D}_{\ell}$ is empty and so $v \in \mathcal{D}_{d / \ell}$. Thus we obtain

$$
\begin{aligned}
\operatorname{card}\left(\mathcal{D}_{\ell, d / \ell} \cap \mathcal{D}_{d / \ell, \ell}\right) & \leq N_{\ell} \frac{1}{q(q-1)} D_{d / \ell} \\
& \left.\leq \frac{1}{q(q-1)}(q-1) q^{\ell} \frac{d}{\ell} q^{\ell+d / \ell^{2}} \quad \text { (end of } \S 5.5 .2\right) \\
& \leq \frac{d}{\ell} q^{d / \ell^{2}+2 \ell-1} .
\end{aligned}
$$

The conclusion follows, as for all $d>6$ we have $d / \ell^{2}+2 \ell-1 \geq 5$.

Acknowledgments. We wish to thank Joachim von zur Gathen for interesting discussions in Lille and in Bonn.

\section{References}

[1] I. V. Arzhantsev and A. P. Petravchuk, Closed polynomials and saturated subalgebras of polynomial algebras, Ukraïn. Mat. Zh. 59 (2007), 1783-1790.

[2] A. Bodin, Reducibility of rational fractions in several variables, Israel J. Math. 164 (2008), 333-348. 
[3] L. Busé, G. Chèze and S. Najib, Noether's forms for the study of non-composite rational functions and their spectrum, preprint.

[4] G. Chèze and S. Najib, Indecomposable polynomials via jacobian matrix, preprint.

[5] A. Dujella and I. Gusić, Indecomposability of polynomials and related diophantine equations, Quart. J. Math. Oxford Ser. 498 (2005), 173-199.

[6] M. Fried and M. Jarden, Field Arithmetic, Ergeb. Math. Grenzgeb. 11, Springer, 2004.

[7] J. von zur Gathen, Counting reducible and singular bivariate polynomials, Finite Fields Appl. 14 (2008), 944-978.

[8] -, Counting decomposable multivariate polynomials, preprint.

[9] —, Counting decomposable univariate polynomials, preprint.

[10] D. Lorenzini, Reducibility of polynomials in two variables, J. Algebra 156 (1993), $65-75$.

[11] S. Najib, Une généralisation de l'inégalité de Stein-Lorenzini, ibid. 292 (2005), 566-573.

[12] A. Schinzel, Polynomials with Special Regard to Reducibility, Encyclopedia Math. Appl. 77, Cambridge Univ. Press, 2000.

[13] Y. Stein, The total reducibility order of a polynomial in two variables, Israel J. Math. 68 (1989), 109-122.

[14] U. Zannier, On the reduction modulo $p$ of an absolutely irreducible polynomial $f(x, y)$, Arch. Math. (Basel) 68 (1997), 129-138.

Laboratoire Paul Painlevé, Mathématiques

Université Lille 1

59655 Villeneuve d'Ascq Cedex, France

E-mail: Arnaud.Bodin@math.univ-lille1.fr

Pierre.Debes@math.univ-lille1.fr

Salah.Najib@math.univ-lille1.fr 